\title{
ANP-AHP Supported Group Decision Support System for Public and Private Sector Participation in New Delhi Expressway System
}

\author{
Madhuri \\ Department of Mathematics \\ G.L.N.A Institute of Technology, \\ Mathura, India
}

\author{
P. K. Saksena \\ Retired Dean and Professor \\ Faculty of Engineering \\ Dayalbagh Educational Institute, Agra, India
}

\begin{abstract}
Delhiite's love affair with the automobile is becoming even more 'passionate' and inspite of the authorities trying desperately hard, the traffic gridlock merely moves on to the next intersection. The complexity of the goals of New Delhi Transport System and its relationship with other Public and Private Organizations makes the managerial decision making regarding New Delhi Expressway System Planning, a multicriteria, multi-dimensional and multi-approach task. It is not realistic to submit decisions to such a critical problem without proper visualization of the preferred future. In this paper, a ANP-AHP based Group Decision Support System for Information Visualization is presented and its main components are analyzed and discussed for effective managerial decision making by mutual participation of public and private sector in New Delhi Expressway System to solve the city's traffic problems. The Model Base Management Subsystem interacts with Project Planning and Implementation Module, Project Financing and Managing Module, Project Infrastructure Leasing Module, Subsidy and Pricing Module; Multi-criteria Iterative Futuristic Decision Process Methodology based Multi-criteria Futuristic Decision Process Platform and Quality Decision Network-Virtual Desk through Central Vision Navigator Board for strategic, tactical and operational planning of New Delhi Expressway System for the next 5 years.
\end{abstract}

\section{Keywords}

New Delhi Expressway System, Analytic Network Process, Analytic Hierarchy Process, Group Decision Support System, Information Visualization.

\section{INTRODUCTION}

It is clear that cars, taxis, two-wheelers and buses form the backbone of the Transport System in New Delhi. This has led to traffic jams and traffic gridlock a very common scene and thus overall travel time and pollution has gone several folds. Sarna (1990) reviewed the traffic and transportation problems of New Delhi and suggested the need of modification of urban transportation planning process, keeping in view the socioeconomic environment prevailing in the country. Khanna, Singh and Vrat (1987) used system dynamics methodology to model the passenger transportation system of New Delhi Urban Region, with a view to analyze some transportation system management policies.
Reddy, Bhatia and Suthar (1990) indicated significant traffic growth and changing composition of traffic flow on selected arterial of New Delhi. Parida and Gupta (1996) presented a logical development and application of a stated preference methodology for mode choice analysis. The authors considered only work trips, since in New Delhi work trips influence the peak hour traffic flow conditions significantly. The results of stated preference modeling were also compared with the conventional revealed preference modeling to demonstrate the relative merits of both the approaches. Sibal (1996) studied the available funding sources for urban mass transit projects in the developing countries and suggested an approach to urban transport financing under the socioeconomic conditions prevalent in India.

In New Delhi the condition of public bus transport i.e. Delhi Transport Corporation (DTC) remains far from adequate. The road has already reached its saturation capacity of 110,000 vehicles per day. The vision is either to go up or go down. An elevated road over the existing ring road has been visualized, as the most feasible solution in the present circumstances. Since there will be no signals in this expressway, it will speed up traffic considerably and the cost will be paid by collecting toll from the users.

It is a globally recognized fact while dealing with such direct public effecting policies as expressway, an announced measure can be delayed or even revoked after a brief implementation due to opposition from the pressure-groups, or a measure may be modified according to people's expectations. Sometimes it also happens that any new measure promulgated may not be implemented. This is what that happened in our capital city. The case of construction of approximately twenty five fly-over in the city in about last three years testifies to this and the problems that were faced by the general public in the form of traffic diversions, jams and that by the authorities in getting clearance from different government departments for land and infrastructure acquirement.

The most acceptable explanation for the cause of the problems faced in the past is that the problem solving approach was based on single criteria whereas it was a multi-criteria, multidimensional and multi-approach managerial problem and so it did not yield satisfactory results. 
Following the present trends of booming vehicular traffic in our Indian mega-cities, the day is not far off when such New Delhi Expressway project will have necessarily to be implemented in New Delhi as well as other Indian MegaCities. There is a dire need to design a support system for implementing New Delhi Expressway Project and then to take its help in managerial decision making regarding policy design and implementation for other Indian mega-cities so that haphazard situations like in New Delhi fly-over project do not take place in the near future. This has led researchers to focus on the quality based Information Visualization in the design of Group Decision Support Systems (GDSS) for New Delhi Expressway Project which facilitates solution of such an unstructured problem by a set of decision-makers working together as a group.

In this paper a ANP-AHP based Group Decision Support System for New Delhi Expressway System (NHGDSSNES) is designed which helps in managerial decision making for the next five years by comparing the effectiveness of various alternate policies with the objective of reducing traffic problems, high infrastructure cost and improving effective coordination among various organizations and providing better commuter facility. Here the policy implementation is dealt with four somewhat different overlapping zones viz. Project Planning and Implementation Module, Project Financing and Managing Module, Project Infrastructure Leasing Module, Subsidy and Pricing Module. The multicriteria and futrologistic approach of Vision Technology Base (VTB) comprising of Multi-criteria Iterative Futuristic Decision Process (MIFDP) Methodology based Multi-criteria Futuristic Decision Process (MFDP) Platform and Quality Decision Network (QDN)-Virtual Desk provides an effective dimension to deal with such complex matters as of Policy Planning and Delivery for New Delhi Expressway System. Moreover, the Information Visualization Support of Central Vision Navigator Board (CVNB) and user friendly support of Dialogue Management System (DMS) makes the NHGDSSNES take decisions and answer 'what if' queries very effectively.

The proposed NHGDSSNES based on Vision Technology Base (VTB) addresses real life complex futuristic problems of New Delhi Expressway System. It allows the decision-makers to lay out a problem as they see it in its complexity and to redefine its definition and structure through interaction and thus to locate futuristic decision priorities. Through a mathematical sequence, it synthesizes their futuristic judgements into an overall estimate of relative futuristic priorities of alternative courses of action and evaluates their effectiveness. NHGDSSNES tracks inconsistencies into the decision-makers judgements and preferences thereby enabling them to assess the Quality of their futuristic assessment knowledge and the stability of the solution.

\section{GROUP DECISION MAKING PROCESS}

A group decision making process also known as an Electronic Meeting System (EMS) is a set of software, hardware, language components and procedures that support a group of people engaged in a decision related meeting and its major function is to support the three common group activities retrieval, sharing and use of information.

The formulation of an overall policy, goals, planning, coordination, balancing, prioritizing different initiatives and actions regarding New Delhi Expressway System and following the reviewing of action plans from an overall perspective can only be done by bringing both the technological, beaurocratic fraternity and private operators at a common platform, thus bridging the existing gap and facilitating effective managerial decision making.

The main factor in a group decision making, is the need for interaction between the group decision members during the decision making process to focus on quality based information visualization. Lim, Raman and Kwok-Kee (1991) have studied the effects of GDSS on decision making in various contexts, involving important variables such as group task and group size. Tavana, Kennedy and Joglekar (1996) have presented a framework to help a group of decision makers define and articulate a hierarchy of hiring criteria and subcriteria and rate each of the candidates on that hierarchy. Hatcher (1992) presented a design of a geographically distributed group decision system that was used by the US Army.

Teng and Ramamurthy (1993) proposed a functional taxonomy on two dimensions, content support and process support. The validity of the taxonomy was then demonstrated by fitting a large number of implemented or proposed systems in GDSS research onto one of the 16 types. Saini, Saxena and Kalra (2000) described the application of internet technology for efficient management of the operational organizations for creation of synergistic intelligent group decision making and support systems.

There is a great need for expertise sharing between group decision members which is very much essential when a complex decision problem like New Delhi Expressway System is to be divided into hierarchy of sub-decision problems, each of which is then solved by individual expert teams within the group for effective decision-making.

\section{DESCRIPTION OF ANP-AHP BASED GROUP DECISION SUPPORT SYSTEM}

Group Decision Support System NHGDSSNES has been designed and developed with symbiotic approach to achieve the aim of providing the necessary quality based information visualization and processing, for real life interaction and speedy decision management to public works department, New-Delhi municipal corporation, state government of Delhi and other concerned authorities by scanning the future.

NHGDSSNES is comprised of five main components - Model Base Management Subsystem (MBMS), Data Base Management Subsystem (DBMS), Central Vision Navigator Board (CVNB), Vision Technology Base (VTB) and Dialog Management Subsystem (DMS). The proposed NHGDSSNES is based on a Central Vision Navigator Board problem solving architecture, where Vision Technology Base comprised of Multi-criteria Iterative Futuristic Decision Process methodology (Saxena Arti and Saxena 2003) based Multicriteria Futuristic Decision Process (MFDP) Platform and ANP-AHP based Quality Decision Network-Virtual Desk associated to different New Delhi Expressway application modules interact through a Central Vision Navigator Board. The basic components of the NHGDSSNES architecture are presented in Fig. 1. It underlines the main modules relating to the Project Planning and Implementation (PPI), Project Financing and Managing (PFM), Project Infrastructure Leasing (PIL), Subsidy and Pricing (SAP). Each module is 
customized with Data Base Management Subsystem (DBMS) and Vision Technology Base (VTB).

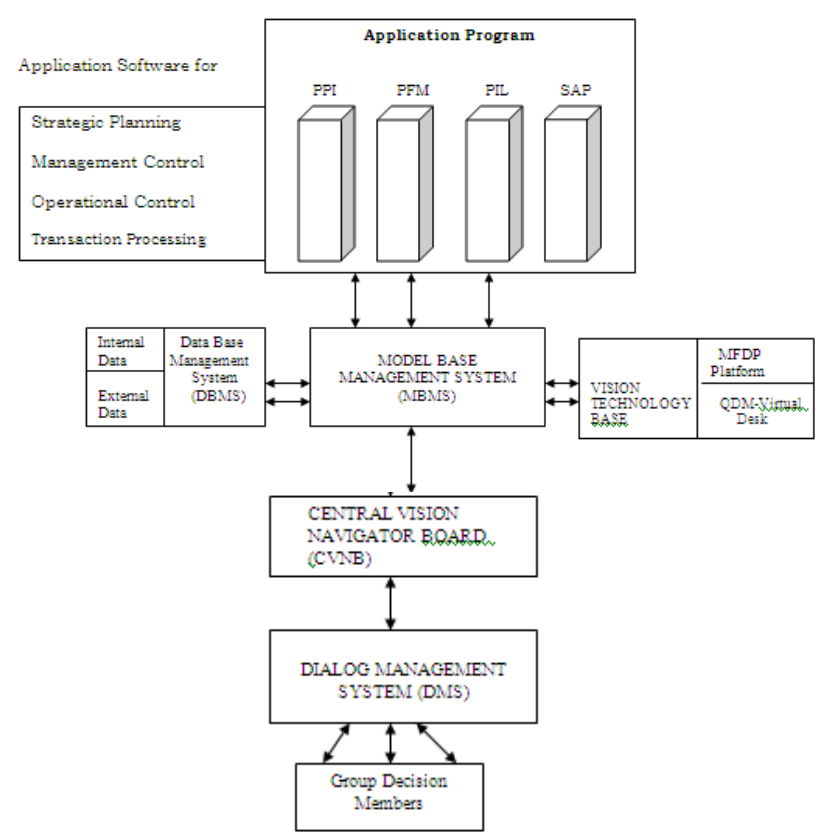

Fig1: Architecture of NHGDSSNES

The interaction between the group decision members, expressway experts the world over and the global database takes place through a MFDP Platform and Quality Decision Network-Virtual Desk for strategic planning, management control, operational planning and transaction processing with the tasks of defining, scanning, managing and prioritizing the Multi Futuristic Decision Indicators. The group facilitator coordinates the group use of VTB and DMS is used by both the group facilitator and the group decision members. The source code of NHGDSSNES is written in 'Visual Basic'. The system has many special features, which are very essential in a well-designed software package. Fig 2 depicts the opening screen of NHGDSSNES.

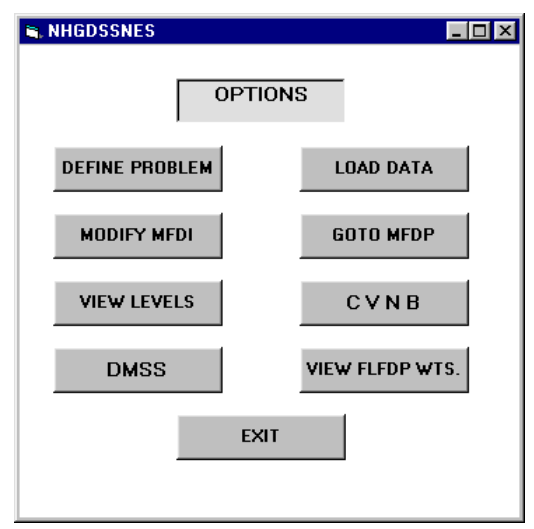

Fig 2: Opening Screen of NHGDSSNES

\section{MODEL BASE MANAGEMENT SUBSYSTEM}

The Model Base Management Subsystem interacts with VTB comprising of Multi-criteria Iterative Futuristic Decision Process methodology based MFDP Platform and QDNVirtual Desk; Project Planning and Implementation (PPI) Module, Project Financing and Managing (PFM) Module, Project Infrastructure Leasing (PIL) Module and Subsidy and Pricing (SAP) Module; and Data Base Management Subsystem through CVNB for strategic planning, management control, operational planning and transaction processing.

\section{MULTI-CRITERIA FUTURISTIC DECISION PROCESS PLATFORM}

MFDP is a information visualization and group decisionranking platform based on feedback of multi disciplinary functional teams of New Delhi Expressway decision making team, New Delhi Expressway expert team and interdisciplinary future scan team to simulate New Delhi Expressway decision problems. MFDP Platform is primarily based on following main principles:

- The New Delhi Expressway current decision plan for the goal is developed by New Delhi Expressway expert team with the aid of the possible pictures of the future, characterized as- a negative picture, a neutral picture and an optimistic picture.

- The New Delhi Expressway current decision plan generated by Outside - In Approach and Inside - Out Approach is clustered into Multi Futuristic Decision Indictors by New Delhi Expressway expert team.

MFDP Module allows groups to lay out a complex decision problem as they see it in its complexity and to redefine its definition and structure through interaction.

\subsection{M.I.F.D.P. Methodology}

MFDP Platform is based on MIFDP methodology which is a multi-disciplinary, multi-dimensional, multi-person, multiobjective, multi-level, multi-period methodology and it implicates iterative multi-criteria approach for futurologistic decision problems and consolidates information about tangible and intangible criteria and alternatives in futuristic managerial decision making process. MIFDP methodology is a non-linear framework based on analytic hierarchic process (Saaty 2000) for analyzing both deductive and inductive iterative futuristic thinking that allows the consideration of several Multi Futuristic Decision Indicators at a time, along with a iterative feedback control mechanism and numerical trade-off without the use of the syllogism. The steps of MIFDP methodology are:

Step 1: Formulation of problem and goal by Decision Making Team (DMT)

Step 2: Formation of interdisciplinary future scan team and expert team from different related areas and fields by DMT.

Step 3: Generation of Current Decision Plan (CDP) by expert team to meet the goal, by Seth-Harva method.

Step 4: Cluster formation of CDP by DMT into a responsible set of mutually exclusive and encompassing ' $i$, Multi Futuristic Decision Indicators (MFDI).

Step 5: Preparation of handout about the goal and delphi technique for interdisciplinary future scan team by DMT. 
Step 6: Design and development of Delphi Questionnaire (DPQ) for Interdisciplinary Future Scan Team by Decision-Making Team.

Step 7: Derivation and analysis of Futuristic Controlled Feedback (FCF) Weights $f_{i}^{r}(r=$ Delphi Rounds) by Decision-Making Team, applying repeated rating and ranking of ' $i$ ' MFDI for different levels $(L=1,2,3, \ldots, l)$ by Interdisciplinary Future Scan Team.

Step 8: If the degree of stability is there, then go to step 9, otherwise go to step 7.

Step 9: Formation of Input Satisfaction Response Questionnaire (ISRQ) from different MFDI and their respective $f_{i}^{r}$ for Interdisciplinary Future Scan Team by Decision-Making Team.

Step10: Satisfaction and dissatisfaction response by Interdisciplinary Future Scan Team for $f_{i}^{r}$.

Step11: Computation of Futuristic Satisfaction Response (FSR) Weights $s_{i}$ by Decision-Making Team using analysis of satisfaction response.

Step12: Computation of Prioritized Futuristic Satisfaction (PFS) Weights $P_{j}$ for prioritized MFDI $j$.

Step13: Calculation of Futuristic Relative Comparison (FRC) Weights $w_{j k}^{\prime}$.

$$
\begin{array}{cc}
w_{j k}^{\prime}=0, & \text { if } j>k \\
w_{j k}^{\prime}=\frac{P_{k} \times 100}{P_{j}}, & \text { if } j \leq k
\end{array}
$$

Step14: Formation of Futuristic Relative Calibration (FRC) Matrix $\quad F^{\prime}\left(n_{L} \times n_{L}\right), \quad$ consisting of $\left[n_{L}\left(n_{L}-1\right)\right] / 2$ futuristic judgments, by calibrating $w_{j k}^{\prime}$ from Futuristic Calibrating Chart.

Step15: Preparation of Futuristic Pairwise Comparison Questionnaire (FPCQ) from $j$ for Expert Team by Decision-Making Team.

Step16: Generation of Futuristic Pairwise Judgments (FPJ) Matrix $F^{\prime \prime}\left(n_{L} \times n_{L}\right)$ consisting of $\left[n_{L}\left(n_{L}-1\right)\right] / 2$ futuristic judgments from FPCQ using Relative Importance Scale (Saaty 1994) by Expert Team.

Step17: Generation of Average Futuristic Relative Judgment (AFRJ) Matrix $F$ from Matrix $F^{\prime}$ and Matrix $F^{\prime \prime}$ for different $L$

Step18: Computation of Futuristic Eigen Vectors (FEV), Futuristic Consistency Index (FCI) and Futuristic Consistency Ratio (FCR) by Analytic Hierarchic Process for all generated Matrix $F$.

Step19: Generation of Futuristic Decision Priority (FDP) Weights $w_{j}^{f L}$ for all $L$ of the problem.

For proper Information Visualization of New Delhi Expressway System, five members of the NDEDMT selected IFS Team consisting of 387 members (136 from North Delhi, 92 from South Delhi, 63 from East Delhi, 57 from West Delhi and 39 Floating Population of Delhi) and NDEET consisting of total 36 Experts (Team I: 5 Futurologists; Team II: 12 Transportation System Experts from Ministry of Transport, New Delhi; Central Road Research Institute (C.R.R.I.), Delhi; Delhi Transport Corporation (D.T.C.), New Delhi; Team III: 12 Operation Research Researchers from Delhi University,
Delhi; I.I.T. Delhi; Institute of Urban Transport, New Delhi, Indian Statistical Institute (I.S.I.), New Delhi; Operation Research Society of India (O.R.S.I) Members and Team IV: 7 Experienced Managers from RITES Limited. New Delhi; Engineers India Limited (E.I.L.). A brief summary of Goal with sufficient technical data, related to New Delhi Expressway System was prepared by NDEDMT. The optative picture, created by NDEET, for effective Managerial Decision Making of New Delhi Expressway System for the next five years, generated total 148 MFDI for different levels. DPQ was formed from generated MFDI for New Delhi Expressway System. MFDIs of Goal were rated and ranked by IFS Team. Feedback of IFS Team was analyzed to compute Futuristic Decision Priority (FDP) Weights $w_{j}^{f L}$ for the Goal.

\section{QUALITY DECISION NETWORK - VIRTUAL DESK}

The ANP-AHP based Quality Decision Network (QDN)Virtual Desk receives its data input from MFDI based on Futuristic Decision Priority (FDP) Weights $w_{j}^{f L}$. The job of understanding the variability in the process is probabilistic and based on the experiences and suggestions of the QDN Virtual Team which aims to preserve the best practice but eliminate the necessity of physical co-location thus enabling the team to collaborate rapidly and continuously with Infrastructure Suppliers and Transportation Managers worldwide, irrespective of the Geographical constraints.

The QDN- Virtual Desk is provided with modern multimedia and networking technologies in order to achieve high levels of mutual affinity and fast decision making in a virtual meeting environment. This provides a totally new level of cognitivestyle thinking in Multi Criteria Decision Making and Group Decision Making. Advances in hardware and software are serving as catalysts in the working of Virtual Teams and therefore QDN - Virtual Desk adds another important dimension in GDSS to generate an excellent virtual meeting environment.

The QDN-Virtual Team achieves the highest level of customer contentment subject to organizational constraints on cost, time and other resources. The working methodology adopted by QDN-Virtual Desk is:

Step1: Selection of Prioritized Multi Futuristic Decision Indicators (MFDI) based on Futuristic Decision Priority (FDP) Weights $w_{j}^{f L}$ using MFDP Platform.

Step2: Selection of Prioritized Target Population ' $p$ ' using MFDP Platform for Target Population.

Step3: Formation of Target Population Evaluation (TPE) Chart for different levels $(L=1,2,3, \ldots, l)$

a) Define the Present Environment for the ' $p$ ' prioritized Target Population and Competitors of the Goal.

b) Define the Strategic Vision of the Goal for the respective Target Population keeping in mind the prioritized MFDI.

c) Calculate the Futurologistic Improvement Vision Factor (FIVF), Futurologistic Weighing Vision Ratio (FWVR) and Futurologistic Normalized Vision Weights (FNVW) for the respective Target Population. 
d) Develop Comparison Matrix between Target Population and prioritized MFDI ' $j$ ' (Askin and Dawson 2000).

e) Calculate the Overall Futuristic Decision Priority (OFDP) Weights $W_{j}^{f L}$ for prioritized MFDI ' $j$ ' using Futurologistic Normalized Score (FNS) and FDP Weights $w_{j}^{f L}$ for each Target Population.

Step4: Select the most prioritized MFDI and formulate Matrix $W_{T P}^{f L}$ for levels $(L=1,2,3, \ldots, l)$ using Overall Futuristic Decision Priority (OFDP) Weights $W_{j}^{f L}$ of the concerned MFDI.

$$
\text { For } L=1, \quad W_{1}^{f L}=W_{T P}^{f L}
$$

Step5: Using Analytic Network Process (Saaty 2001), formulate the Clusters for levels $(L=1,2,3, \ldots, l)$ of the most prioritized MFDI. These MFDI are mutually inter-connected according to their Outer Dependence (influencing or being influenced).

Step 6: Taking at a time one Cluster in mind Go to Step 13 to Step 19 of MFDP Platform for the other connected Clusters influencing this one and calculate Futuristic Decision Priority (FDP) Weights $w_{j}^{f L}(L=1,2,3, \ldots, l)$ for this cluster.

Step 7: Repeat Step 6 for each Cluster at the level.

Step 8: Formulate Futuristic Cluster (FC) Matrix $W_{2}^{f L}(L=1,2,3, \ldots, l)$.

Step 9: Repeat Steps 5 to Step 8 to formulate Futuristic Cluster (FC) Matrix $W_{3}^{f L+1}(L=1,2,3, \ldots, l)$.

Step 10: Formulate Futuristic Level Relationship (FLR) Matrix $W_{4}^{f L, f L+1}(L=1,2,3, \ldots, l)$ between two consecutive hierarchical levels from their respective Futuristic Decision Priority (FDP) Weights $w_{j}^{f L}$.

Step 11: Frame Futuristic Quality Super (FQS) Matrix $M$ :

$$
\begin{aligned}
& \text { Goal } \\
& L+1
\end{aligned}\left[\begin{array}{lcl}
0 & 0 & 0 \\
W_{1}^{f L} & W_{2}^{f L} & W_{1}^{f L} \\
0 & W_{4}^{f L, f L+1} & 0
\end{array}\right]
$$

and make it Column Stochastic. The Final Limit Futuristic Decision Priority (FLFDP) Weights $W_{j}^{S P^{*}}$ priorities are obtained by $\operatorname{raising} M$ to $\lim _{z \rightarrow \infty} M^{2 z+1}$.

Step12: Select Super Prioritized FLFDP Weights $W_{j}^{S P^{* *}}$ for different levels $(L=1,2,3, \ldots, l)$ of MFDI.

$$
\text { or } L=2,3, \ldots, l, \quad W_{1}^{f L}=\left(W_{T P}^{f L}+W_{j}^{S P^{* *}}\right) / 2 \text {. }
$$

Step13: Selection of the Optimal Super Prioritized Final Limit Futuristic Decision Indicator Scenarios and Action Plan for the Goal which are forwarded to the Central Vision Navigator Board (CVNB) for effective display of the action plan to be considered by the Group Decision Members (GDM).

\section{PROJECT PLANNING AND IMPLEMENTATION MODULE (PPI)}

\subsection{Advantages}

- The PPI module helps in the formation of organization, structure to better define the goals and devise a organizational solution which results in the ongoing analysis and implementation of New Delhi Expressway transit.

- This module also facilitates the policy formation for Decision Making and Management for New Delhi Expressway. The detail content of policy includes all specific priority measures. It also deals with Planning and Implementation of Transport Engineering Factors.

\subsection{PPI Decisions}

The module helps the decision-makers in following decision regarding New Delhi Expressway issues:

$\rightarrow$ Will the system be flexible enough, affordable, selfenforcement type and if there is any scope for incremental development?

$\rightarrow$ Will the existing system provide necessary facilities like (Property land for Bus terminals and Stops / Smooth Driving Conditions for Transit Vehicles)?

$\rightarrow$ Is the designed New Delhi Expressway capable of adjusting to Heavy Transit flows?

$\rightarrow$ Is there any delay experienced by Transit Vehicles and if yes what is the Cost of Delay and how to solve the problem?

\section{PROJECT FINANCING AND MANAGING (PFM) MODULE}

Large projects such as New Delhi Expressway are beyond the Financial and Managerial capacity of the public sector. Therefore, it is necessary to involve private sector and joint venture in a big way in large projects of transport development.

\subsection{Advantages}

- The PFM module deals with and defines the role of both the Private and Public Sector Partners in the project and limits the Area of Work. Public Sector role as Facilitator / Coordinator / Controller and Private sector as Doer / Implementers.

- The module provides the Group Decision Members (GDM) with comprehensive Physical and Financial Plans including Project Reports.

\subsection{PFM Decisions}

The module helps the GDM in following type of Decisions:

$\rightarrow$ How and by whom Compensation is to be provided to Economically Weaker Sections (EWS) due to Land-use?

$\rightarrow$ What Methods to be adopted for the Reduction of Cost given to Private Operators (Subsidies /Commercial and Trucking Activity /Retail Commercial Spaces/ Infrastructure Facility)?

$\rightarrow$ How the Developmental Plans for the project to be financed (Fare Hike/ Betterment Levy/ Additional Taxes)? 


\section{PROJECT INFRASTRUCTURE LEASING (PIL) MODULE}

The cost of improvement needed to develop New Delhi Expressway Infrastructure to acceptable levels of service requires huge amount of budgetary resources. So, it has become necessary to resort to private sector participation in creation and operation of such big projects as this New Delhi Expressway.

\subsection{Advantages}

- The PIL module analyzes all the prospects regarding BOT (Build, Operate, and Transfer) concept, risks and returns between the two sectors and thus promotes fair negotiations and meaningful decisions.

- The module provides a Promising Strategy to channelise private initiatives towards National Development. It leads to an Understanding of Private and Government Operators on Equitable Devices of Risk Sharing.

- The PIL module varies the parameters linked to certain decision issues and then simulates the effect of that variation on the New Delhi Expressway project. For example the project viability from the point of view of NPV, IRR, \& ROE are found to be highly sensitive to change in construction period.

\subsection{PIL Decisions}

$\rightarrow$ What are the Risk and Returns in the project for Private and Public Sectors?

$\rightarrow$ How is the Cost-Benefit Analysis (A Public Sector Concern)?

$\rightarrow$ How much are the Performance Parameters like IRR, ROE, NPV etc. (A Private Sector Concern)?

\section{Subsidy and Pricing (SAP) Module}

The objective of New Delhi transport system to provide cheap and affordable service conflicts with the financial principles of running a system without incurring losses brings the concept of Subsidy and Pricing in picture in planning of the New Delhi Expressway Project.

\subsection{Advantages}

- The SAP module looks whether the project is not only economic or uneconomic but also provides values to beneficiaries other than riders willing to support the system.

- The module helps in decisions regarding the Fairness and Equity understanding among both Private and Public Sectors, which is further divided into Actual Transport Condition Policy, Policy Design and Development.

\subsection{SAP Decisions}

$\rightarrow$ The basic question answered is 'Who is paying and Who is benefiting?'

$\rightarrow$ How is the Accessibility of the System distributed to different Social Groups and Classes?

$\rightarrow$ What should be the Physical Performance indicators for granting subsidy to private operators? (Equipment Productivity/ Staff Productivity / Fuel Efficiency /Time Consumed)?

\section{DATA BASE MANAGEMENT SUBSYSTEM}

NHGDSSNES utilizes a relational DBMS for reducing computation time and effort for supportive repetitive interaction processes. The main task of the DBMS is the simplification, preparation, and pre-processing of input data of New Delhi Expressway System and also control and verification of the bulk of data required by the application programs. The relational database, which is comprised of daily transport analysis report as reported by DTC after preprocessing provides reliable data for necessary policy framework. The pre-processing of this data involves separation of transport analysis data, data of traffic jams and gridlock, bad roads and traffic engineering policy and further does the job of cleansing, structuring and formatting of data to maintain data integrity in DBMS. MS EXCEL electronic spreadsheet, which is linked to relational database, facilitates post processing of records returned by the database, which is extensively and intensively queried through structured query language (SQL).

The relational DBMS is comprised of the following fields:

- Subsidies, Equity and Shares

- Technical Specifications

- Finance (Methods and Sources)

- Tax and Toll Structure

- Traffic Engineering Facilities

- Vehicle Operating Cost

- Pollution Cost

- Infrastructure \& Expressway Status

The relational DBMS is two-dimensional and Group Decision Members (GDM) interacts with tables that have lines and columns like a spreadsheet and can easily travel through the data by the use of full screen editor that permits them to scroll the data in all directions.

\section{CENTRAL VISION NAVIGATOR BOARD (CVNB)}

The designed NHGDSSNES is based on a Central Vision Navigator Board Problem Solving Architecture where MFDP Platform and ANP-AHP based Quality Decision Network (QDN)-Virtual Desk based Vision Technology Base connected to various New Delhi Expressway System components interact through a Central Vision Navigator Board (CVNB). In this CVNB common globally shared database, local knowledge base and specialized Vision Technology Base sources act upon a Central Vision Navigator Board Problem Solving Architecture, according to a strategy aiming at building a group problem solution, both cooperatively and opportunistically.

The Central Vision Navigator Board for New Delhi Expressway System is based on independent and interactive knowledge based agents as Data Completion, Data Analysis, Information Visualization, and Prediction and Control functions. It stores a representation of the New Delhi Expressway System structures and services and provides facility for viewing animated generation of prioritized course of action chart in real time as a result of simulation. It attracts the attention of the Group Decision Members (GDM) and its visual presentation assist cognition and early decision making. The Central Vision Navigator Board is well suited for realtime systems and is basically communication medium that enables information to be shared among the GDM. The 
modular and distributed computing environment allows a kind of parallel processing and reasoning with the integration of Vision Technology Base (VTB) and Group Decision Members (GDM).

Central Vision Navigator Board supports new data types and provides synchronization of multiple data streams and real life applications via real time scheduling and fast interrupt processing. The merger of the discrete media like tables and text, with the continuos media such as audio and images make the New Delhi Expressway System Information Visualization and Interaction Process more direct and straight for Group Decision Members (GDM), improves retention, and this plays a pivotal role in the positive contribution to the support system.

\section{DIALOG MANAGEMENT SUBSYSTEM}

The Dialogue Management System (DMS) is designed for Group Decision Members (GDM) with a variety of New Delhi Expressway System decision-making needs. The GDM are able to select their area of interest Module (PPI / PFM / PIL / SAP). The DMS captures the GDM's preferences, degree of expertise, skills and then interprets the Group Decision Member's input, which is conveyed to Central Vision Navigator Board (CVNB) and finally presents the result in the form of charts, text, graphs and tables along with suitable form and visual displays. The DMS capabilities of NHGDSSNES are broadly classified into two categories: Decision Support Display Desk and Queries Support Display Desk due to variety of users with different decision making tasks. While Queries Support Display Desk allows adhoc retrieval of New Delhi Expressway System Information, Decision Support Display Desk supports the New Delhi Expressway System decision-making tasks and allows the user to generate a number of displays from the data available in the system, into pre-defined format.

\section{CONCLUSION}

An ANP-AHP based Group Decision Support System for Information Visualization of New Delhi Expressway System (NHGDSSNES) is presented and its main components are analyzed and discussed. In particular emphasis is focused on the Data Base Management Subsystem, Model Base Management Subsystem, Multi-criteria Iterative Futuristic Decision Process (MIFDP) Methodology based Multi-criteria Futuristic Decision Process Platform and ANP-AHP based Quality Decision Network-Virtual Desk based Vision Technology Base, Central Vision Navigator Board and Dialog Management Subsystem. Vision Technology Base provides a comprehensive framework for group decision making of multi-criteria Complex decision problems of New Delhi Expressway System for the next five years by choosing the best one in a set of competing futuristic alternatives. NHGDSSNES facilitates method that allows Group Decision Members to make managerial decision by combining futuristic judgements and personal values in a logical way. It solves ill-structured Expressway System Management problems and incorporates the informed futuristic judgement, assumption and intuition of experienced decision members. The system incorporates the best of formal, incremental and systematic paradigms and accounts for dynamic interaction among Decision-members, proceeding logically from New Delhi Expressway Current Decision Plan through performance criteria to alternative decisions or mixes of scenarios and action plan. The designed System can test existing as well as new plans and several "What-if" situations and evaluate the associated impacts. The NHGDSSNES will help in operating at strategic, tactical and operational level, through the best use of the existing facilities.

\section{RERENCES}

[1] Askin, R.G., and Dawson, D.W. 2000. Maximizing Customer Satisfaction by Optimal Specification of Engineering Characteristics. IIE Transactions 32, 9-20.

[2] Hatcher, M.E. 1992. A Video Conferencing System for the United States Army: Group Decision- Making in a Geographically Distributed Environment. Decision Support Systems 8, 181-190.

[3] Khanna, I.K., Singh, N., and Vrat, P. 1987. Some Studies in Policy Design and Analysis of Delhi's Urban Passenger Transportation System. Transportation System Management and System Analysis and Policy Studies. Tata McGraw-Hill.

[4] Lim, L., Raman, K., and Kwok-Kee, W. 1991. Interacting effects of GDSS and Leadership. Decision Support Systems 12, 199-211.

[5] Parida, N., and Gupta, A.K. 1996. A Stated Preference Approach to Mode Choice Modeling in Delhi. Transports urbains dans les pays en developpement, actes de la conference Tome 1, III.131-III.139.

[6] Reddy, T.S., Bhatia, N.L., and Suthar, H.H. 1990. Traffic Flow Characteristics of Selected Arterials in Delhi. Transportation Systems Studies: Analysis and Policy. Papyrus Publishing House.

[7] Saini, R., Saxena, P.K., and Kalra, P.K. 2000. Internet Enabled Synergistic Intelligent Systems and their Applications to Efficient Management of Operational Organizations. Information Sciences 127, 45-62.

[8] Sarna, A.C. 1990. Traffic and Transportation Problems of Indian Cities-A Review. Transportation System Studies: Analysis and Policy. Papyrus Publishing House.

[9] Saxena, A., and Saxena, P.K. 2003. Future Scan and Prospective Vision of Delhi Bus Transportation System Using Multicriteria Iterative Futuristic Decision Process Methodology. Urban Transport Journal 4(1), 208-213.

[10] Sibal, V.K., 1996. Financing Urban Mass Transit Projects in India: with a special referenced to the proposed Delhi MRTS. Transports urbains dans less pays en developpement, actes de la conference Tome 1, II.161-11.170.

[11] Tavana, M., Kennedy, D.T., and Joglekar, P. 1996. A Group Decision Support Framework for Consensus Ranking of Technical Manger Candidate. Omega 24(5), 523-538.

[12] Teng, J., and Ramamurthy, K. 1993. Group Decision Support Systems: Clarifying the Concept and Establishing a Functional Taxonomy. INFOR 31(3), 166184. 